\title{
MiR-144-3p is associated with pathological inflammation in patients infected with Mycobacteroides abscessus
}

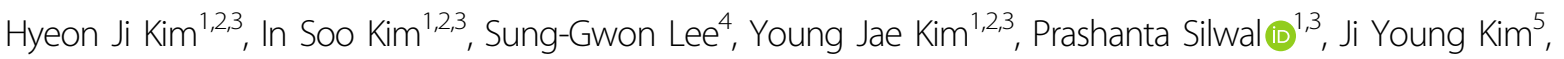 \\ Jin Kyung Kim ${ }^{1,3}$, Wonhyoung Seo ${ }^{1,2,3}$, Chaeuk Chung ${ }^{3,6}$, Hyun Kyu Cho ${ }^{7}$, Hee Jae Huh ${ }^{8}$, Seung Cheol Shim, \\ Chungoo Park ${ }^{4}$, Byung Woo Jhun ${ }^{7}$ and Eun-Kyeong Jo (i)
}

\begin{abstract}
Infection with rapidly growing nontuberculous mycobacteria is emerging as a global health issue; however, key host factors remain elusive. Here, we investigated the characteristic immune profiles of peripheral blood mononuclear cells (PBMCs) from patients infected with Mycobacteroides abscessus subsp. abscessus (Mabc) and M. abscessus subsp. massiliense (Mmass). Using an integrated analysis of global mRNA and microRNA expression profiles, we found that several inflammatory cytokines/chemokines [interleukin (IL)-1 $\beta, I L-6, C-X-C$ motif chemokine ligand 2 , and C-C motif chemokine ligand 2] and miR-144-3p were significantly upregulated in PBMCs from patients compared with those from healthy controls (HCs). Notably, there was a strong correlation between the expression levels of miR-144-3p and proinflammatory cytokines/chemokines. Similarly, upregulated expression of miR-144-3p and proinflammatory cytokines/chemokines was found in macrophages and lungs from mice after infection with Mabc and Mmass. We showed that the expression of negative regulators of inflammation (SARM1 and TNIP3) was significantly downregulated in PBMCs from the patients, although they were not putative targets of miR-144-3p. Furthermore, overexpression of miR-144-3p led to a marked increase in proinflammatory cytokines/chemokines and promoted bacterial growth in macrophages. Together, our results highlight the importance of miR-144-3p linking to pathological inflammation during M. abscessus infection.
\end{abstract}

\section{Introduction}

There is growing concern over the increasing incidence and prevalence of nontuberculous mycobacteria (NTM) infection worldwide $^{1}$. Among NTM, Mycobacteroides abscessus - which includes $M$. abscessus subsp. abscessus (Mabc), M. abscessus subsp. massiliense (Mmass), and $M$. abscessus subsp. bolletii-exhibits rapid growth. $M$. abscessus is clinically significant because of its multidrug

Correspondence: Chungoo Park (chungoo.park@gmail.com) or Byung Woo Jhun (byungwoo.jhun@gmail.com) or Eun-Kyeong Jo (hayoungj@cnu.ac.kr) ${ }^{1}$ Department of Microbiology, Chungnam National University School of Medicine, Daejeon 35015, Korea

${ }^{2}$ Department of Medical Science, Chungnam National University School of Medicine, Daejeon 35015, Korea

Full list of author information is available at the end of the article

These authors contributed equally: Hyeon Ji Kim, In Soo Kim, Sung-Gwon Lee resistance and the limited efficacy of the available treatments ${ }^{2-5}$. The risk of M. abscessus infection is associated with immune deficiency, immunosuppressive therapy, old age, and underlying lung and systemic diseases ${ }^{6,7}$. However, the host immune factors by which protection and pathological responses are modulated during M. abscessus infection are unclear.

The host immune response is a determinant of the outcome of NTM infection because most NTM strains are ubiquitous and not contagious. While several cytokines, including tumor necrosis factor (TNF) and interleukin (IL)-32, have potential as biomarkers for certain NTM diseases ${ }^{6-8}$, our understanding of the nature and regulation of the immune response to NTM infection is incomplete. In addition, innate immune signaling is

\section{(c) The Author(s) 2021}

(c) (i) Open Access This article is licensed under a Creative Commons Attribution 4.0 International License, which permits use, sharing, adaptation, distribution and reproduction in any medium or format, as long as you give appropriate credit to the original author(s) and the source, provide a link to the Creative Commons license, and indicate if changes were made. The images or other third party material in this article are included in the article's Creative Commons license, unless indicated otherwise in a credit line to the material. If material is not included in the article's Creative Commons license and your intended use is not permitted by statutory regulation or exceeds the permitted use, you will need to obtain permission directly from the copyright holder. To view a copy of this license, visit http://creativecommons.org/licenses/by/4.0/. 
tightly regulated to ensure effective immunity and prevent damage by excessive inflammation ${ }^{9,10}$. Thus, it is important for us to identify and quantify key host factors that influence the balance between pathological inflammation and protective immune responses during NTM infection.

MicroRNAs (miRNAs), small noncoding RNA molecules, are critical post-transcriptional regulators of immune and inflammatory responses ${ }^{11}$. MiRNAs also have potential as biomarkers of various infectious diseases ${ }^{12,13}$. Evaluation of the profile and function of differentially expressed miRNAs in NTM patients will enable the development of biomarkers and enhance our understanding of immunopathology during NTM infection.

Here, we sought to investigate the immune mRNA and miRNA signature of peripheral blood mononuclear cells (PBMCs) from untreated pulmonary disease patients infected with Mabc or Mmass. Several proinflammatory cytokines/chemokines were significantly upregulated in PBMCs from the NTM patients compared with those from the healthy controls (HCs). In addition, miR-144-3p levels were significantly upregulated in PBMCs from the patients and in murine bone marrow-derived macrophages (BMDMs) after infection with Mabc or Mmass or in infected lung tissues from mice. Further study showed that the levels of SARM1 (sterile $\alpha$ and TIR motifcontaining 1 protein) ${ }^{14}$ and TNIP3 (TNFAIP3 interacting protein 3$)^{15}$, negative regulators of inflammation, were depressed in PBMCs from the patients compared with those from the HCs, although they were not putative targets of miR-144-3p. Furthermore, overexpression of miR-144-3p led to a marked increase in inflammatory cytokines/chemokines and increased bacterial growth in BMDMs. Together, these data suggest that pulmonary disease patients with $M$. abscessus infection had increased pathological inflammation through upregulation of $m i R$ 144-3p.

\section{Materials and methods}

\section{Ethics statement}

Clinical and laboratory data of study patients were obtained from an ongoing Institutional Review Board-approved prospective observational cohort study to investigate NTM pulmonary disease (clinicaltrials.gov identifier NCT00970801). This study was approved by the Institutional Research and Ethics Committee at Chungnam National University Hospital (CNUH 2019-04-046; Daejeon, Korea).

\section{NTM identification}

Sputum was obtained for microbiological evaluation. Acid-fast bacilli smears and cultures were conducted using standard methods. NTM species were identified using polymerase chain reaction (PCR)-reverse blot hybridization assay (REBA) targeting the $r p o B$ gene or via nested multiplex PCR-reverse line blot (RLB) hybridization assays of the internal transcribed spacer (ITS) region.

For PCR-REBA targeting of the rpoB gene, a REBA Myco-ID kit (YD Diagnostics, Seoul, South Korea) was used. For this assay, $3 \mu \mathrm{L}$ of template deoxyribonucleic acid (DNA) from clinical isolates was used. The rpoB gene region was amplified with biotin-labeled primers. The PCR conditions were as follows: one cycle at $94{ }^{\circ} \mathrm{C}$ for $5 \mathrm{~min} ; 45$ cycles at $94^{\circ} \mathrm{C}$ for $30 \mathrm{~s}$ and $65^{\circ} \mathrm{C}$ for $30 \mathrm{~s}$; and one cycle at $72{ }^{\circ} \mathrm{C}$ for $7 \mathrm{~min}$. The PCR products were hybridized to a membrane with immobilized probes. The data were interpreted using the REBA Myco-ID data sheet provided by the manufacturer.

For the multiplex PCR-RLB hybridization assay of the ITS region, AdvanSure Mycobacteria GenoBlot Assay (LG Life Sciences, Seoul, South Korea) was used. The AdvanSure assay was performed according to the manufacturer's instructions. Briefly, PCR was performed using $25 \mu \mathrm{L}$ of reaction mixture containing $2 \times$ master mix, $5 \mu \mathrm{L}$ of primer mixture, and $7.5 \mu \mathrm{L}$ of sample DNA. The PCR conditions were as follows: one cycle at $50^{\circ} \mathrm{C}$ for $2 \mathrm{~min}$ and $95^{\circ} \mathrm{C}$ for $10 \mathrm{~min} ; 15$ cycles at $94{ }^{\circ} \mathrm{C}$ for $30 \mathrm{~s}, 65^{\circ} \mathrm{C}$ for $1 \mathrm{~min}$, and $72{ }^{\circ} \mathrm{C}$ for $30 \mathrm{~s} ; 38$ cycles at $94{ }^{\circ} \mathrm{C}$ for $30 \mathrm{~s}, 55^{\circ} \mathrm{C}$ for $1 \mathrm{~min}$, and $72{ }^{\circ} \mathrm{C}$ for $30 \mathrm{~s}$; and one cycle at $72{ }^{\circ} \mathrm{C}$ for $10 \mathrm{~min}$. Hybridization was performed with $20 \mu \mathrm{L}$ of the amplified sample and confirmed by observing blue coloration after adding the streptavidin-alkaline phosphatase conjugate. The color intensity of the band was measured using AdvanSure GenoScan (LG Life Science).

\section{Cell cultures}

Human PBMCs from healthy volunteers were isolated from heparinized venous blood using Ficoll-Hypaque (Lymphoprep; Axis-shield, 1114544, Dundee, Scotland) as described previously ${ }^{16}$. For monocyte-derived macrophage (MDM) differentiation, adherent monocytes were incubated in Roswell Park Memorial Institute 1640 medium (Lonza, 12-702 F, Basel, Switzerland) containing $5 \%$ pooled human serum (Sigma-Aldrich, H4522, MO, USA) and $1 \% \mathrm{~L}$-glutamine for $1 \mathrm{~h}$ at $37^{\circ} \mathrm{C}$, after which the nonadherent cells were removed. Human MDMs were prepared by culturing peripheral blood monocytes for 4 days in the presence of $4 \mathrm{ng} / \mathrm{mL}$ human macrophage colony-stimulating factor (M-CSF) (R\&D, 216-MC, MN, USA).

For preparation of the mouse BMDMs, bone marrow was collected and cultured for 3-5 days in Dulbecco's modified Eagle's medium (Lonza, 12-604F) containing $10 \%$ fetal bovine serum (Serana, S-FBS-US-015, Pessin, Germany) and antibiotics (Lonza, 17-745E) in the presence of M-CSF (R\&D, 416-ML). Cells were incubated in a $37^{\circ} \mathrm{C}$ humidified atmosphere with $5 \%$ carbon dioxide. 


\section{Mice}

In this study, 8- to 12-week-old (age-matched) male C57BL/6 (Samtako Bio, Gyeonggi-do, Korea) mice were used. Mice were maintained on a $12 \mathrm{~h}$ light/dark cycle under specific pathogen-free conditions. All animal experimental methods and procedures were performed following the relevant ethical guidelines and regulations approved by the Institutional Research and Ethics Committee at Chungnam National University, School of Medicine (201912A-CNU-203; Daejeon, Korea) and the guidelines of the Korean Food and Drug Administration.

\section{Nanostring nCounter assay and data analysis}

Nanostring nCounter Human Immunology gene expression assays and Human miRNA expression assays were performed at PhileKorea Technology (Daejeon, South Korea) using the NanoString nCounter GX Human Immunology Kit V2 (NanoString Technologies, Inc., Seattle, WA, USA) and nCounter Human miRNA Assay Kit V3 (NanoString Technologies), respectively. nSolver, version 4.0 (NanoString Technologies), was utilized for differential gene expression analyses from nCounter assay data. Based on the nSolver 4.0 Analysis Software User Manual, all reporter code count files from nCounter instruments passed the default QC settings. All raw gene expression data were normalized in positive control normalization and housekeeping normalization steps by default. Fold changes were computed by taking the mean of the normalized lanes from healthy controls, and false discovery rate (FDR)-adjusted $p$-values were calculated using the Benjamini-Yekutieli procedure.

\section{RNA extraction and quantitative real-time (qRT)-PCR}

Human PBMCs were isolated from heparinized venous blood using Ficoll-Hypaque (Lymphoprep; Axis-shield, 1114544, Dundee, Scotland) following the manufacturer's manual. Total RNA from PBMCs was extracted using QIAzol lysis reagent (Qiagen, 1023537, Hilden, Germany) and miRNeasy Mini Kits (Qiagen, 217004) according to the manufacturer's instructions, followed by RNA quantitation and assessment using QIAxpert (Qiagen).

After RNA quantitation, complementary DNA (cDNA) from total RNA was synthesized using reverse transcription master premix (Elpis Biotech, EBT-1515, Daejeon, South Korea). For miRNA expression analysis, cDNA from total RNA was synthesized using miScript II RT Kits (Qiagen, 218161), as described previously ${ }^{17}$. qRT-PCR was performed in the Rotor-Gene Q 2plex system (Qiagen, 9001620) using SYBR Green master mix (Qiagen, 204076) or miScript SYBR Green PCR Kit (Qiagen, 218073) and primers for the indicated genes. The primer sequences are shown in Supplementary Table 1. Relative expression levels of mRNA and miRNA were calculated with the $2-\Delta \Delta \mathrm{Ct}$ method.

\section{Preparation of mycobacterial strains, infection, and intracellular colony-forming unit (CFU) assays}

Mabc (ATCC 19977) and Mmass (KMRC-00136-13018) were incubated at $37^{\circ} \mathrm{C}$ using an orbital shaker in Middlebrook $7 \mathrm{H} 9$ medium containing $10 \%$ oleic albumin dextrose catalase (BD, Franklin Lakes, NJ, USA) until the mid-log phase $\left(\mathrm{OD}_{600 \mathrm{~nm}}=0.4\right)$, as described previously ${ }^{18}$.

Cells were inoculated for $2 \mathrm{~h}$ with either Mabc or Mmass, washed three times with phosphate-buffered saline (PBS) to remove extracellular bacteria, and incubated in fresh media in a $37^{\circ} \mathrm{C}$ humidified atmosphere with $5 \%$ carbon dioxide. For mouse infection in vivo, mice were administered Mabc or Mmass intranasally (i.n.; $1 \times$ $10^{7} \mathrm{CFU} /$ mouse). For determination of the bacterial burden, mice were euthanized at the indicated times after NTM infection. The lungs were removed and homogenized using a tissue homogenizer (OMNI TH; Omni International, GA, USA) in PBS with $0.1 \%$ Tween ${ }^{\circledR} 20$, as described previously ${ }^{18}$.

For analysis of bacterial viability in host cells by CFU assays, infected cells were lysed in distilled water to release intracellular bacteria. Thereafter, the serially diluted homogenates of the infected tissues or cells were inoculated onto 7H10 agar plates, and colonies were counted 3-4 days later.

\section{Bioinformatics analysis}

For each gene trait, the expression levels from the 24 samples were first transformed on a $\log 2$ scale before principal component analysis (PCA). PCA was performed using the "prcomp" function in the "stats" package in $\mathrm{R}$ (version 3.2.4) ${ }^{19}$ with the first two principal components. We performed in silico analysis to identify putative miRNA target genes using TargetScan Human Release 7.1 (http://www.targetscan.org/) ${ }^{20}$. Functional and pathway enrichment analyses were performed using the DAVID (version 6.8, https://david.ncifcrf.gov) ${ }^{21}$ and PANTHER (version 15.0, http://www.pantherdb.org) ${ }^{22}$ classification systems with a human reference gene set. To visualize volcano plots and heatmaps, we utilized the "plot" function in the "graphics" package in R.

\section{Cell transfection}

The hsa-miR-144-3p and mmu-miR-144-3p mimics (sense 5'-UACAGUAUAGAUGAUGUACU- ${ }^{\prime}$, antisense $5^{\prime}$-AG UACAUCAUCUAUACUGUA-3') were the same and purchased from Genolution (Seoul, South Korea). The mimic negative control (4464058) was purchased from Invitrogen (CA, USA). Oligonucleotides were transfected into BMDMs or human primary MDMs using Lipofectamine 2000 (Invitrogen, 11668019) according to the manufacturer's instructions as described previously ${ }^{16}$. For real-time PCR, an hsamiR-144-3p primer (MS00020328) and mmu-miR-144-3p primer (MS00032326) were purchased from Qiagen. 


\section{Statistical analysis}

Statistical analyses were performed with Analyse-it, version 5.1 (Analyse-it Software, Ltd., Leeds, UK), SPSS Statistics for Windows, version 24.0 (SPSS, Inc., Chicago, IL, USA), and GraphPad Prism, version 5.0 (GraphPad Software, San Diego, CA, USA). The data were processed by principal component analysis, Spearman's correlation, Student's $t$-test, Mann-Whitney $\mathrm{U}$ test, ANOVA, and Kruskal-Wallis test, as appropriate, and are detailed in each figure and figure legend above. The results are presented as the mean \pm SEM (standard error of the mean) and significance $\left(" p<0.05,{ }^{* *} p<0.01,{ }^{* * * *} p<0.001\right.$, ns not significant).

\section{Results}

\section{Clinical characteristics of NTM patients}

The characteristics of the 31 Mabc-infected and 22 Mmass-infected patients are presented in Table 1. The mean ages of the Mabc- and Mmass-infected patients were 59 and 55 years, respectively; approximately threequarters were female and had never smoked. The most common underlying condition was bronchiectasis, followed by previous pulmonary tuberculosis and chronic obstructive pulmonary disease in the Mabc and Mmass groups. None of the patients tested positive for the human immunodeficiency virus. All of the patients had respiratory symptoms, such as cough, sputum, or hemoptysis. On chest computed tomography, $93 \%$ of the Mabcinfected and $86 \%$ of the Mmass-infected patients showed a nodular bronchiectatic form (Supplementary Fig. 1). The mean erythrocyte sedimentation rates were 33 and $31 \mathrm{~mm} / \mathrm{h}$ in the Mabc-infected and Mmass-infected patients, respectively. Sputum acid-fast bacilli smears were positive in $48 \%$ of the Mabc-infected and 36\% of the Mmass-infected patients. There were no significant differences in characteristics between the two patient groups.

\section{Immune transcriptome analysis of the NTM patients and the $\mathrm{HCs}$}

We performed nCounter Human Immunology gene expression assays on PBMCs from the Mabc-infected patients $(n=11)$, the Mmass-infected patients $(n=7)$, and the HCs $(n=6)$ (Fig. 1a, Supplementary Table 2). All subjects were female and of similar ages $(p=0.31$ by Kruskal-Wallis test). The mean age was 61.1 years for the Mabc-infected patients, 59.4 years for the Mmass-infected patients, and 55.2 years for the HCs.

To assess transcriptome similarity among 23 datasets, we performed PCA. The HCs were well separated and clustered, and the other two types of patients were intermingled in the PCA, suggesting that there are few or no significant differences in transcriptome-wide gene expression profiles between the Mabc-infected and
Table 1 Baseline characteristics of study patients at diagnosis.

\begin{tabular}{|c|c|c|c|}
\hline Characteristics & Mabc $(n=31)$ & Mmass $(n=22)$ & $P$-value \\
\hline Age, year & $59( \pm 2)$ & $55( \pm 2)$ & 0.187 \\
\hline Female & $22(71)$ & $16(73)$ & 0.889 \\
\hline Body mass index, $\mathrm{kg} / \mathrm{m}^{2}$ & $20.3( \pm 0.4)$ & $20.9( \pm 0.4)$ & 0.367 \\
\hline Never smoker & $25(81)$ & $19(86)$ & 0.585 \\
\hline \multicolumn{4}{|l|}{ Underlying conditions } \\
\hline Bronchiectasis & $28(90)$ & $20(91)$ & 0.943 \\
\hline $\begin{array}{l}\text { Previous pulmonary } \\
\text { tuberculosis }\end{array}$ & $11(36)$ & $8(36)$ & 0.948 \\
\hline $\begin{array}{l}\text { Chronic obstructive } \\
\text { pulmonary disease }\end{array}$ & $4(13)$ & $1(5)$ & 0.389 \\
\hline Diabetes mellitus & $2(7)$ & $0(0)$ & 0.505 \\
\hline Sinusitis & $0(0)$ & $1(5)$ & 0.415 \\
\hline \multicolumn{4}{|l|}{ Symptoms } \\
\hline Cough & $20(65)$ & $15(68)$ & 0.781 \\
\hline Sputum & $25(81)$ & $17(77)$ & 0.765 \\
\hline Hemoptysis & $7(23)$ & $10(46)$ & 0.079 \\
\hline Type of disease & & & 0.229 \\
\hline $\begin{array}{l}\text { Noncavitary nodular } \\
\text { bronchiectatic }\end{array}$ & $25(80)$ & $13(58)$ & \\
\hline $\begin{array}{l}\text { Cavitary nodular } \\
\text { bronchiectatic }\end{array}$ & $4(13)$ & $6(28)$ & \\
\hline Fibrocavitary & $2(7)$ & $3(14)$ & \\
\hline $\begin{array}{l}\text { Erythrocyte sedimentation } \\
\text { rate, } \mathrm{mm} / \mathrm{h}\end{array}$ & $33( \pm 4)$ & $31( \pm 6)$ & 0.417 \\
\hline C-reactive protein, mg/dl & $0.47( \pm 0.16)$ & $0.57( \pm 0.25)$ & 0.657 \\
\hline Albumin, $\mathrm{g} / \mathrm{dl}$ & $4.3( \pm 0.1)$ & $4.2( \pm 0.10)$ & 0.355 \\
\hline Sputum smear positivity & $15(48)$ & $8(36)$ & 0.415 \\
\hline
\end{tabular}

Data are presented as the mean ( \pm standard error of the mean) or numbers (\%). Mabc $=M$. abscessus subsp. abscessus, Mmass $=M$. abscessus subsp. massiliense.

Mmass-infected patients, but these profiles are clearly distinct from the transcriptome of the HCs (Fig. 1b). Next, we identified 143 and 151 differentially expressed genes (DEGs) in the Mabc and Mmass samples compared to the $\mathrm{HC}$ samples, respectively, using both $t$-tests and fold change (FC) analysis (FDR $<0.05$ and $\mathrm{FC}>2$ ) (Fig. 1c). Of the 109 commonly identified DEGs, 28 DEGs were mainly involved in a cytokine-cytokine receptor interaction, which is the most significantly enriched pathway (FDR $<$ $2.06 \times 10^{-16}$ ) using KEGG (Kyoto Encyclopedia of Genes and Genomes) (Fig. 1d), and almost three-quarters (21 out of 28) of the genes were upregulated in the NTMinfected patients. Notably, nine genes [C-C motif 
a

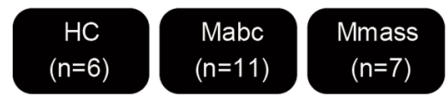

Total RNA isolation

Reporter hybridization

Reporter code count
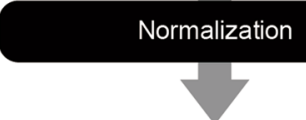

Expression profiles of

579 immune - related genes
C

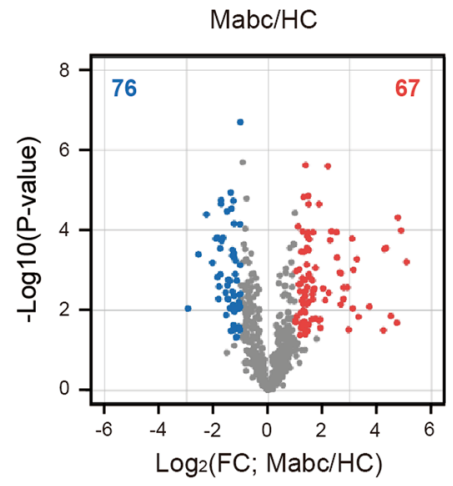

b

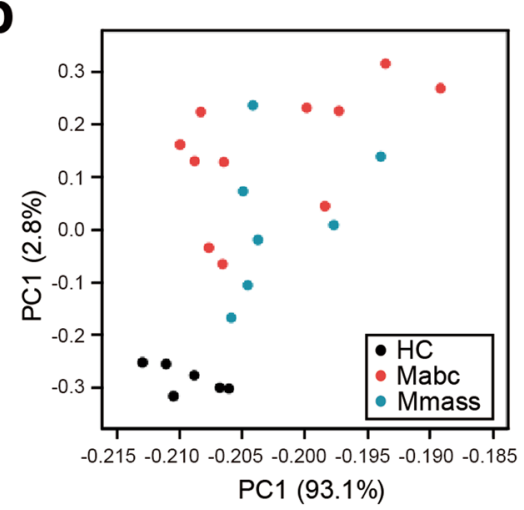

d

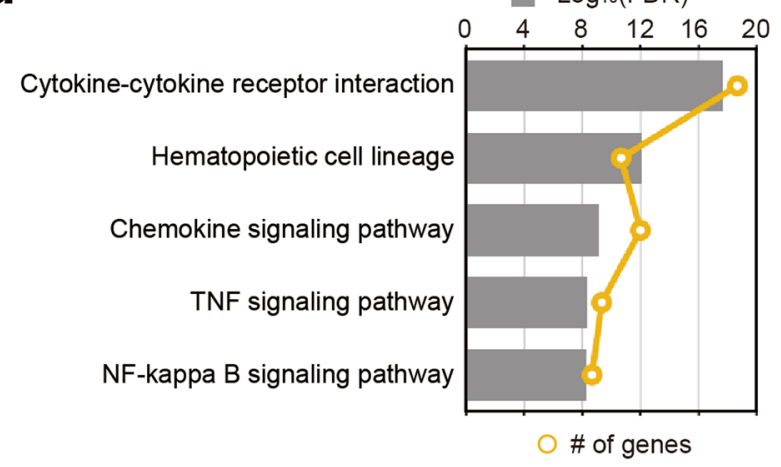

Z-score

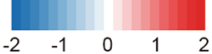

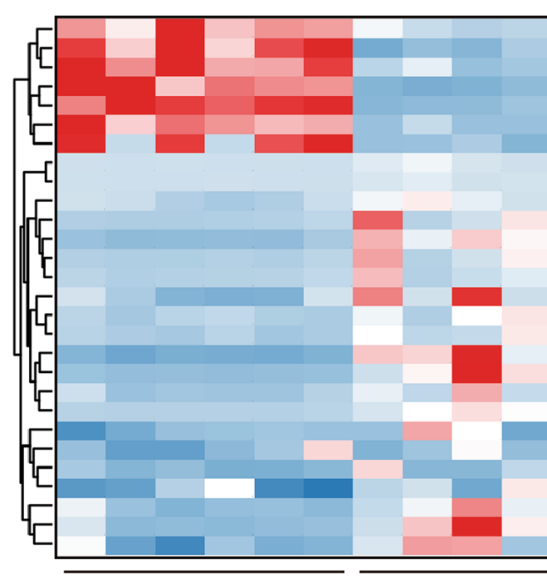

$\mathrm{HC}$

$-2$

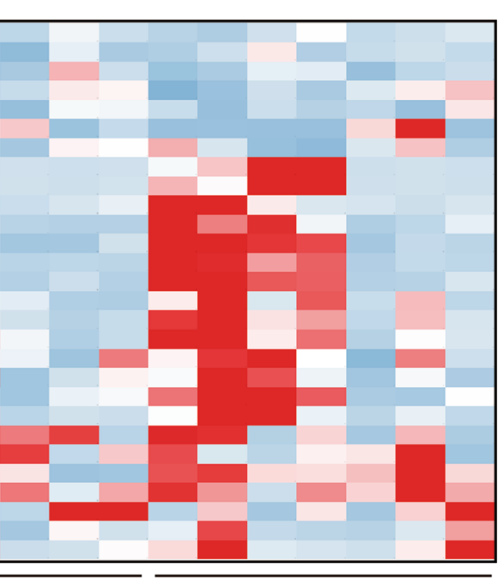

Mmass

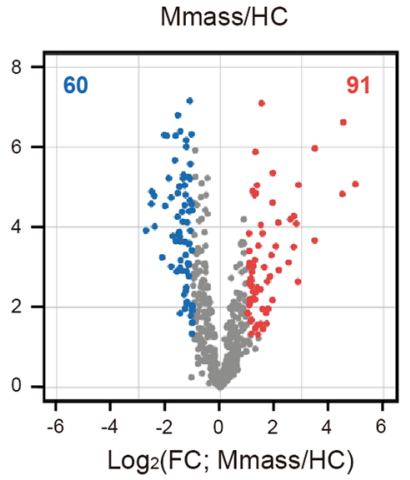

e

Fig. 1 Immune transcriptome analysis reveals differential expression of proinflammatory cytokines/chemokines between PBMCs from the NTM patients and the HCs. a Schematic diagram of the immune transcriptome analysis in this study. b Results of principal component analysis showing the first two principal components (PC1 and PC2) for all tested samples ( $\mathrm{HC}, n=6$; Mabc, $n=11$; Mmass, $n=7$ ). c Volcano plots representing 579 immunerelated genes with the log2-fold change plotted against the negative log10 adjusted $p$-value for the Mabc and Mmass groups compared to the HC groups. Red and blue dots indicate upregulated and downregulated genes compared to the HC group. $\mathbf{d}$ Results of the top 5 KEGG pathways enriched with the 109 common DEGs of the Mabc and Mmass groups. e Heatmap for 28 DEGs that are constituents of the cytokine-cytokine interaction pathway (hsa04060). Nine genes labeled in red indicate that they are upregulated over 10-fold in the Mabc or Mmass group or members of the TNF signaling pathway. An IL-7 gene labeled in blue indicates the most depressed gene in the Mabc and Mmass groups. The hierarchical clustering of genes was performed with Euclidean distance matrices of Z-scores. HC healthy controls, Mabc M. abscessus subsp. abscessus, Mmass M. abscessus subsp. massiliense. 
chemokine ligand (CCL) 2, CCL7, CCL20, CCL22, CCL24, C-X-C motif chemokine ligand (CXCL) 2, CCL24, IL1B, $T N F$, and $I L-6]$ related to immune responses and inflammation were persistently upregulated (over 10-fold) under NTM-infected conditions (Fig. 1e). In addition, $I L$ 7 , a crucial cytokine for T-helper 1 responses ${ }^{23-26}$, was the most downregulated gene, with an $\sim 6$-fold decrease in both types of patients.

\section{Upregulation of proinflammatory cytokines/chemokines in PBMCs from the patients compared with those from the HCs}

To validate the increased expression of cytokines/chemokines, we performed qRT-PCR of PBMCs from the expanded population (Mabc, $n=31$; Mmass, $n=22$; HC, $n=39$ ). The mRNA levels of $I L-1 \beta, I L-6, C X C L 2$, and $C C L 2$ were significantly increased in PBMCs from the Mabc-infected and Mmass-infected patients compared to those from the HCs (Fig. 2a, c-e). The TNF mRNA level was not significantly increased in PBMCs from the Mabcinfected and Mmass-infected patients compared to those from the HCs (Fig. 2b). There were no significant differences in the levels of cytokines/chemokines (IL-1 $\beta, T N F$, $I L-6, C X C L 2$, and CCL2) between the Mabc-infected and Mmass-infected patients. Interestingly, the CCL5 mRNA levels were significantly downregulated in PBMCs from the Mmass-infected patients compared with those from the HCs (Fig. 2f). In addition, the mRNA levels of CCL7, $C C L 20$, and CCL24, but not CCL22, were significantly increased in the patients infected with Mabc or Mmass compared with the HCs (Supplementary Fig. 2).

We then investigated the interrelationships among different cytokines/chemokines in each group (Fig. $2 g-i$ ). In the HCs, there was a significant positive correlation among $I L-1 \beta, I L-6, C X C L 2$, and $C C L 2$, whereas there was a negative correlation between $T N F$ and $C C L 5$ (Fig. $2 \mathrm{~g}$ ). In the Mabc-infected patients, there was a strong positive correlation between $I L-6$ and CXCL2 $(n=31, r=0.543$, $p=0.002), C X C L 2$ and $I L-1 \beta(n=31, r=0.723, p<$ 0.001), CXCL2 and CCL2 $(n=31, r=0.736, p<0.001)$, and $I L-1 \beta$ and $I L-6(n=31, r=0.670, p<0.001)$. However, the TNF levels were negatively correlated with $I L-1 \beta$, $I L-6, C X C L 2$, and CCL2 but not CCL5 (positive correlation; $n=31, r=0.446, p=0.012$ ) (Fig. 2h). In the Mmassinfected patients, there was a strong correlation between $I L-6$ and $I L-1 \beta(n=22, r=0.554, p=0.007), I L-6$ and CXCL2 ( $n=22, r=0.570, p=0.006)$, and CXCL2 and CCL2 $(n=22, r=0.733, p<0.001)$. Similar to the findings observed in the Mabc-infected patients, the TNF levels showed a strong positive correlation with CCL5 in the Mmass-infected patients $(n=22, r=0.560, p=0.007)$ (Fig. 2i). These data suggest an altered relationship of TNF with other proinflammatory cytokines/chemokines in the patients.
We further investigated the relationship between cytokine/chemokine levels and various clinical parameter(s). Each cytokine/chemokine had a strong positive/negative correlation with certain clinical parameter(s) (Supplementary Fig. 3). Notably, in the Mmass-infected patients, the CCL5 level had a strong positive correlation with serum albumin levels $(n=22, \quad r=0.450, p=0.036)$, whereas CXCL2 had a negative correlation with serum albumin levels $(n=22, r=0.418, p=0.053)$. These results suggested that the Mabc-infected and Mmassinfected patients' peripheral immune cells have a distinct set of upregulated proinflammatory cytokines/chemokines, which might be associated with the altered regulation of specific clinical parameter(s).

\section{The miR-144-3p level is upregulated in PBMCs from the patients}

To evaluate the mechanisms underlying the altered proinflammatory cytokine/chemokine expression in the patients, we performed an nCounter Human miRNA Expression Assay of PBMCs and evaluated the miRNA expression levels in the same 23 nCounter samples (Fig. 3a, Supplementary Table 3). We identified 25 (14 upregulated and 11 downregulated) and 10 (8 upregulated and 2 downregulated) miRNAs that were differentially expressed (FDR $<0.05$ and fold change $>2$ ) in the Mabc-infected and Mmass-infected patients, respectively, compared to the HCs (Fig. 3b). Among them, nine (7 upregulated and 2 downregulated) miRNAs had the same expression pattern (Fig. 3c, d). Next, to examine whether these differentially expressed miRNAs are capable of regulating proinflammatory response genes, we screened for potential miRNA target genes using the TargetScan database and then identified significantly (FDR $<5 \%$ ) enriched pathways for the predicted target genes. We found 11, 9, and 23 significant PANTHER pathways in the target genes of $m i R-144-3 p$, miR-1-3p, and $m i R-132-3 p$, respectively (Supplementary Table 4). There were five shared pathways (gonadotropin-releasing hormone receptor pathway; P06664, angiogenesis; P00005, FGF signaling pathway; P00021, EGF receptor signaling pathway; P00018, and CCKR signaling map; P06959), with three immune response-related pathways (interferon-gamma signaling pathway; P00035, T-cell activation; P00053, and interleukin signaling pathway; P00036) (Fig. 3e).

We selected three miRNAs that showed the greatest difference in expression between the patients and the HCs and confirmed that the $m i R-144-3 p$ level was significantly higher in the Mabc-infected and Mmass-infected patients than in the HCs (Fig. 3f; $p<0.001$ ). The levels of the other two miRNAs ( $h s a-m i R-1255 a$ and $h s a-m i R-5001-3 p$ ) were not significantly different (Fig. $3 g$ ). We also examined whether the severity of the condition and the initiation of antibiotic treatment affected the expression of miR-144- 


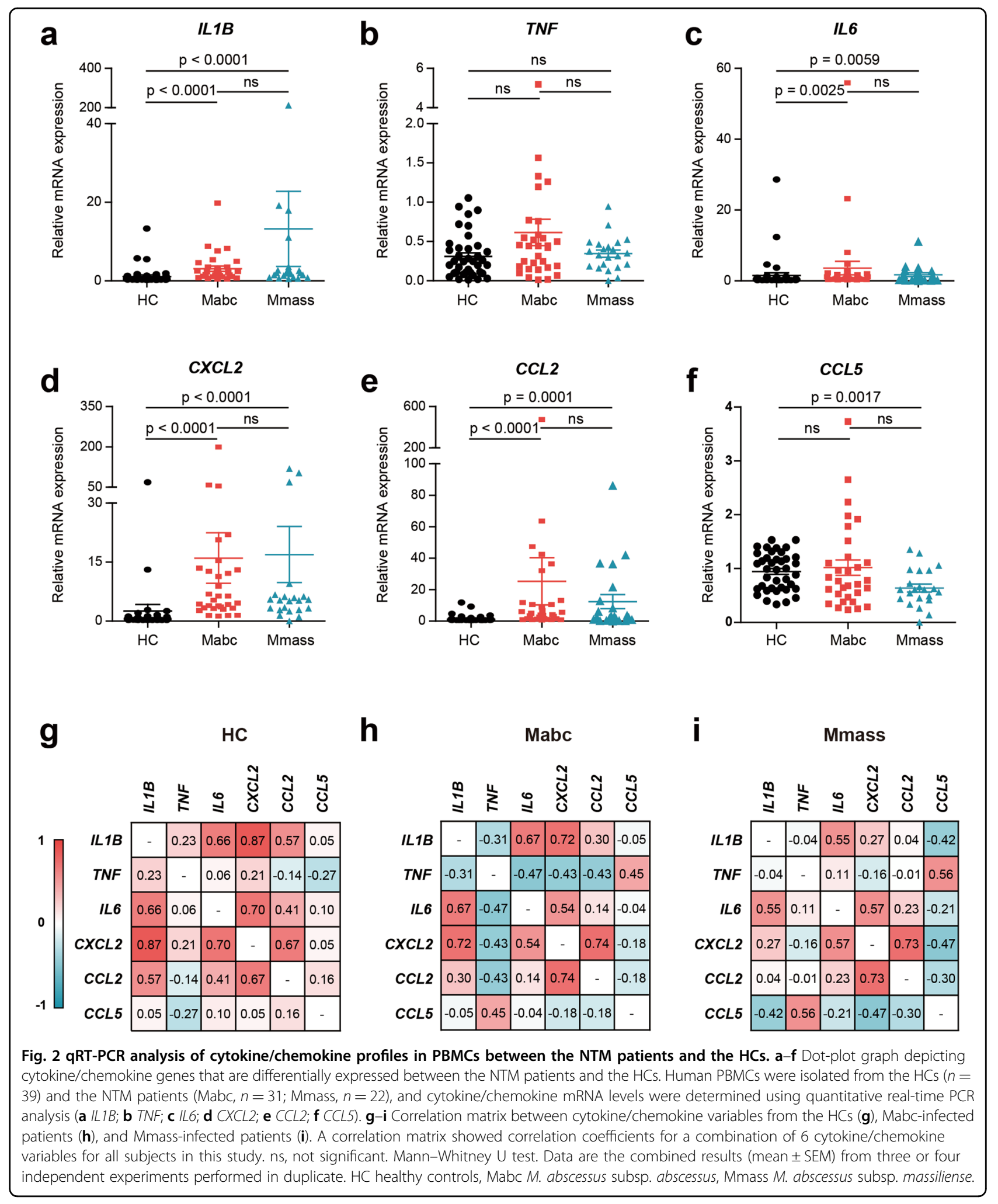

$3 p$. When the severity was divided according to the BACES score ${ }^{27}$ (Supplementary Fig. 4a) and the corresponding expression levels of miR-144-3p were compared, no significant difference was found (Supplementary Fig. $4 \mathrm{~b} ; p=0.905)$. The level of miR-144-3p also did not show any significant difference depending on whether antibiotic 


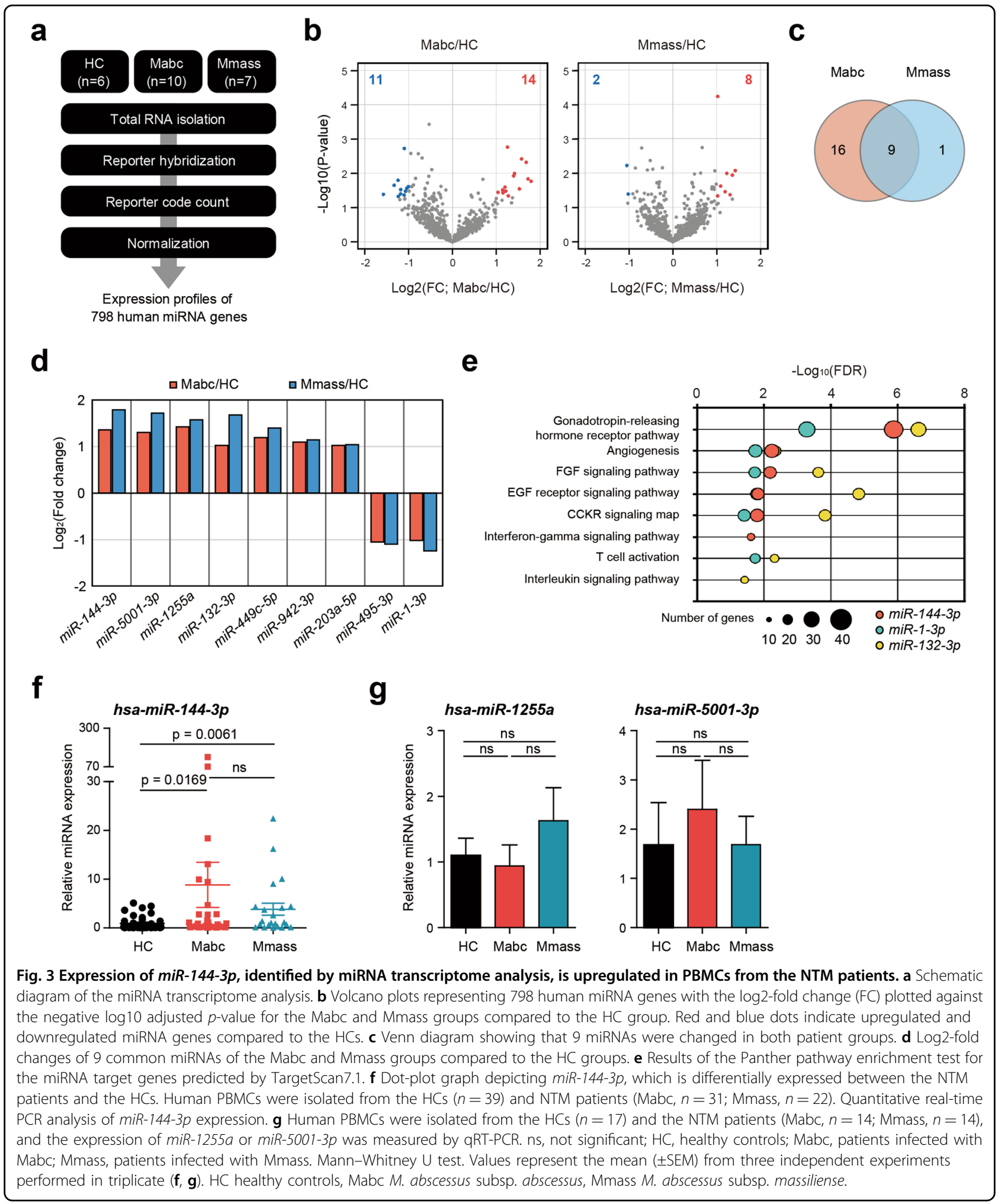

treatment was started (Supplementary Fig. 4c; $p=0.215$ ). Altogether, these data suggested that $m i R-144-3 p$ is a signature miRNA of PBMCs from patients with Mabc or Mmass infection.
MiR-144-3p overexpression increases proinflammatory cytokine/chemokine expression in human macrophages

Hsa-mir-144-3p reportedly plays a proinflammatory role in atopic dermatitis ${ }^{28}$. We thus assessed the 

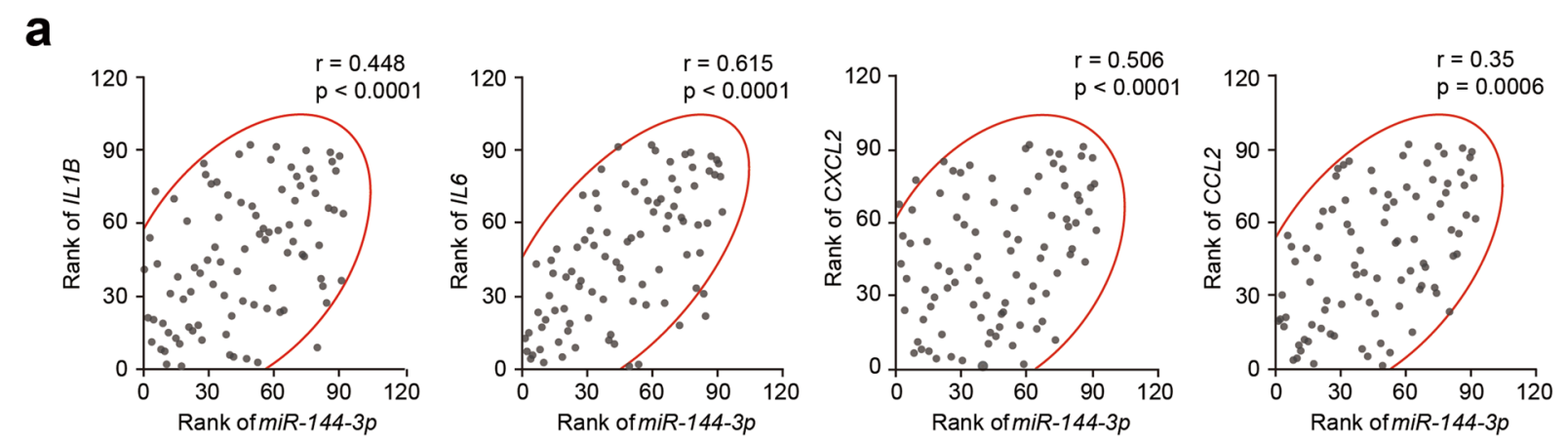

b
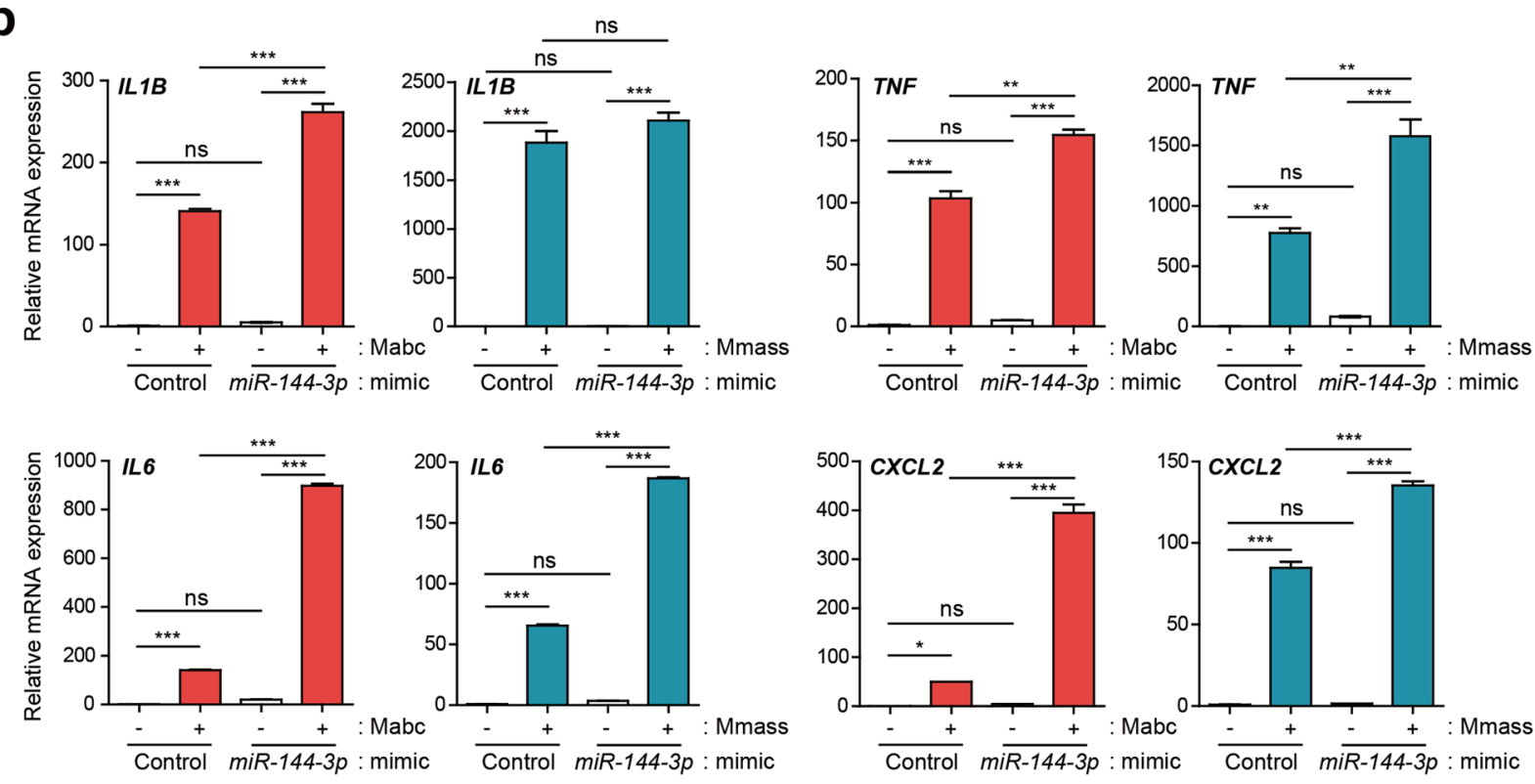

Fig. 4 Overexpression of $\mathbf{m i R}-144-3 p$ upregulates proinflammatory cytokine/chemokine expression in human MDMs. a Scatter plots with $90 \%$ density ellipses showing the rank of miR-144-3p plotted against IL 1B, IL6, CXCL2, and CCL2 for all subjects (HC, $n=39 ;$ Mabc, $n=31 ;$ Mmass, $n=$ 22). Spearman's rank correlation determined the coefficient ( $r$ ) and significance. $\mathbf{b}$ Human primary MDMs were transfected with mimic negative control (Control) or miR-144-3p mimic (10 nM) for $24 \mathrm{~h}$ and then infected with or without Mabc or $\mathrm{Mmass}$ (MOI =2, for each; for $3 \mathrm{~h}$ ). Quantitative real-time PCR analysis of $I L 1 B, I L 6, T N F$, and CXCL2 expression. ${ }^{*} p<0.05$, ${ }^{* *} p<0.01$, and ${ }^{* * *} p<0.001$. Spearman correlation (a); one-way ANOVA (b). Values represent the mean ( $\pm \mathrm{SD}$ ) from three independent experiments performed in duplicate (b). HC healthy controls, Mabc M. abscessus subsp. abscessus, Mmass M. abscessus subsp. massiliense.

relationship between the miR-144-3p level and inflammatory cytokines/chemokines. The miR-144-3p level was significantly positively correlated with those of $I L-1 \beta$ ( $n=$ 92, $r=0.448, p<0.0001), \quad I L-6 \quad(n=92, r=0.615, p<$ 0.0001), CXCL2 ( $n=92, r=0.506, p<0.0001)$, and CCL2 ( $n=92, r=0.35, p=0.0006$ ) (Fig. 4a).

We next transfected human primary MDMs with a miR-144-3p mimic and examined the mRNA levels of $I L$ $1 \beta, I L-6, T N F$, and $C X C L 2$. The overexpression of $m i R-$ 144-3p resulted in significant upregulation of the levels of the four proinflammatory cytokines/chemokines in MDMs infected with Mabc (Fig. 4b). In Mmass infection, miR-144-3p overexpression led to increased $I L-6, T N F$, and $C X C L 2$ levels but did not affect the level of $I L-1 \beta$
(Fig. 4b). The transfection efficiency of $m i R-144-3 p$ is shown in Supplementary Figure 5. Therefore, miR-144-3p induces the mRNA synthesis of proinflammatory cytokines/chemokines in human MDMs following Mabc or Mmass infection.

\section{The levels of the TLR negative regulators SARM1 and TNIP3 are downregulated in PBMCs from patients}

To identify the targets of miR-144-3p in modulating host inflammatory responses, we used the computational target prediction tools miRanda (http://www.microrna. org/microrna/home.do), TargetScan, and DIANA-microT (http://diana.cslab.ece.ntua.gr/microT/). Bioinformatic analysis identified two putative TLR negative regulator 
genes encoding SARM1 ${ }^{14}$ and TNIP $3^{15}$ as $m i R-144-3 p$ targets. We then examined the mRNA expression levels in PBMCs by qRT-PCR analysis and found that both SARM1 and TNIP3 levels were significantly downregulated in both the Mabc $(n=22)$ and Mmass $(n=11)$ groups (Fig. $5 \mathrm{a}, \mathrm{b})$. Furthermore, the SARM1 levels were negatively correlated with the CXCL2 and CCL2 levels in all tested subjects. Similar to these results, the TNIP3 mRNA levels showed an inverse correlation with the CXCL2 and CCL2 levels in all tested subjects (Fig. 5c, d).

To further determine whether miR-144-3p is involved in the downregulation of both SARM1 and TNIP3, we overexpressed human MDMs with a miR-144-3p mimic and assessed the mRNA expression of SARM1 and TNIP3 after Mabc and Mmass infection. The data showed that the overexpression of $m i R-144-3 p$ failed to downregulate and significantly upregulated the mRNA expression of TNIP3 and SARM1 in human MDMs before and after infection with Mabc or Mmass (Fig. 5e). These data revealed that both SARM1 and TNIP3 are not putative targets for $m i R-144-3 p$. Nevertheless, these data suggest that the depressed levels of the TLR negative regulators SARM1 and TNIP3 play a role in the upregulated inflammatory responses in NTM patients.

\section{MiR-144-3p is upregulated in a mouse model in vitro and in vivo}

Because the $m i R-144-3 p$ sequence is identical in humans and mice (Fig. 6a), we next evaluated the effects of Mabc and Mmass infection on the miR-144-3p level in vivo and in vitro. To this end, C57BL/6 mice were infected with Mabc or Mmass for 3 days, and the lungs were harvested for determination of the miRNA and mRNA levels. The mmu-miR-144-3p level was significantly upregulated in the mouse lung after Mabc or Mmass infection (Fig. 6b), as were the levels of the five proinflammatory cytokines and chemokines (Fig. 6c).

We also determined the miRNA and mRNA levels in murine bone marrow-derived macrophages (BMDMs) after Mabc or Mmass infection. Although the peak time point differed between the Mabc and Mmass groups, either strain significantly upregulated the $m m u-m i R-144-$ $3 p$ level in BMDMs (Fig. 6d). In addition, Mabc infection robustly increased the mRNA levels of $T N F, I L-1 \beta$, and CXCL2 in BMDMs in a time-dependent manner (Supplementary Fig. 6a). Interestingly, Mmass infection significantly increased the TNF, $I L-1 \beta$, and CXCL2 levels in BMDMs after $18 \mathrm{~h}$ of infection (Supplementary Fig. 6b). Furthermore, $m m u-m i R-144-3 p$ overexpression led to a slight but significant upregulation of intracellular Mabc and Mmass growth in BMDMs (Fig. 6e). Therefore, the $m m u-m i R-144-3 p$ level is upregulated by Mabc and Mmass infection in vitro and in vivo and contributes to favoring the intracellular growth of Mabc and Mmass.

\section{Discussion}

Compared with Mycobacterium tuberculosis (TB), the causal pathogen of tuberculosis, less is known about the host immunologic factors important during NTM infection, particularly with rapidly growing $M$. abscessus, such as Mabc, Mmass, and M. abscessus subsp. bolletii ${ }^{29,30} . M$. abscessus causes opportunistic pulmonary infections in high-risk patients, including those with cystic fibrosis and bronchiectasis $^{1,29,31,32}$, and can cause pulmonary, skin, and soft tissue infections even in immunocompetent subjects $^{1,29,31,32}$. Importantly, it is emerging as a clinically important strain with an increasing prevalence of antibiotic resistance ${ }^{33}$, although the response rates to antibiotic therapy are somewhat different among subspecies $^{34}$. A mechanistic understanding of the immune profile of NTM disease is needed to develop host-directed therapeutics; however, the key immune biomarkers for patients with Mabc and Mmass infection remain illdefined.

A bioinformatics analysis yielded a large set of proinflammatory cytokines/chemokines elevated in patients and identified several candidate genes for validation in the expanded patient group. The $I L-1 \beta, I L-6, C X C L 2$, and CCL2 mRNA levels were upregulated in the Mabcinfected and Mmass-infected patients compared with the HCs. Our data partly correlate with recent findings that both $M$. abscessus and Mycolicibacterium smegmatis induce high chemokine levels in human monocytic THP1 cells $^{35}$. Notably, there was a strong positive correlation among proinflammatory cytokines/chemokines, except $T N F$, in $M$. abscessus-infected patients. Previous studies using animal models showed that $M$. abscessus-induced $T N F$ is crucial for protective immunity ${ }^{36,37}$ or disease pathology depending on the strain ${ }^{38}$. Despite this, little is known about the expression of TNF in PBMCs between patients infected with Mabc and Mmass. Our data showed that the TNF levels did not differ among PBMCs from all groups. Previous data showed that TNF expression was lower in cultured human monocytes from NTM patients infected with Mycobacterium avium or M. abscessus ${ }^{39}$. In addition, we found that the chemokine CCL5 was depressed in the Mmass-infected patients. CCL5, also known as RANTES (regulated on activation, normal $\mathrm{T}$ cell expressed and secreted), is important for T-cell migration $^{40}$. It was shown that depressed CCL5 in a pleural site of tuberculous pleurisy patients is associated with poor antigenic responses at the disease sites ${ }^{41}$. Together, CCL5 levels may show potential immunobiologic relevance in anti-NTM immunity, particularly against Mmass infection. Interestingly, CCL5 levels showed a strong positive correlation with serum albumin levels in the Mmassinfected patients. Human NTM diseases are extremely diverse, particularly in clinical and microbiological spectrum, geographic distribution, etc ${ }^{31}$. Therefore, the 


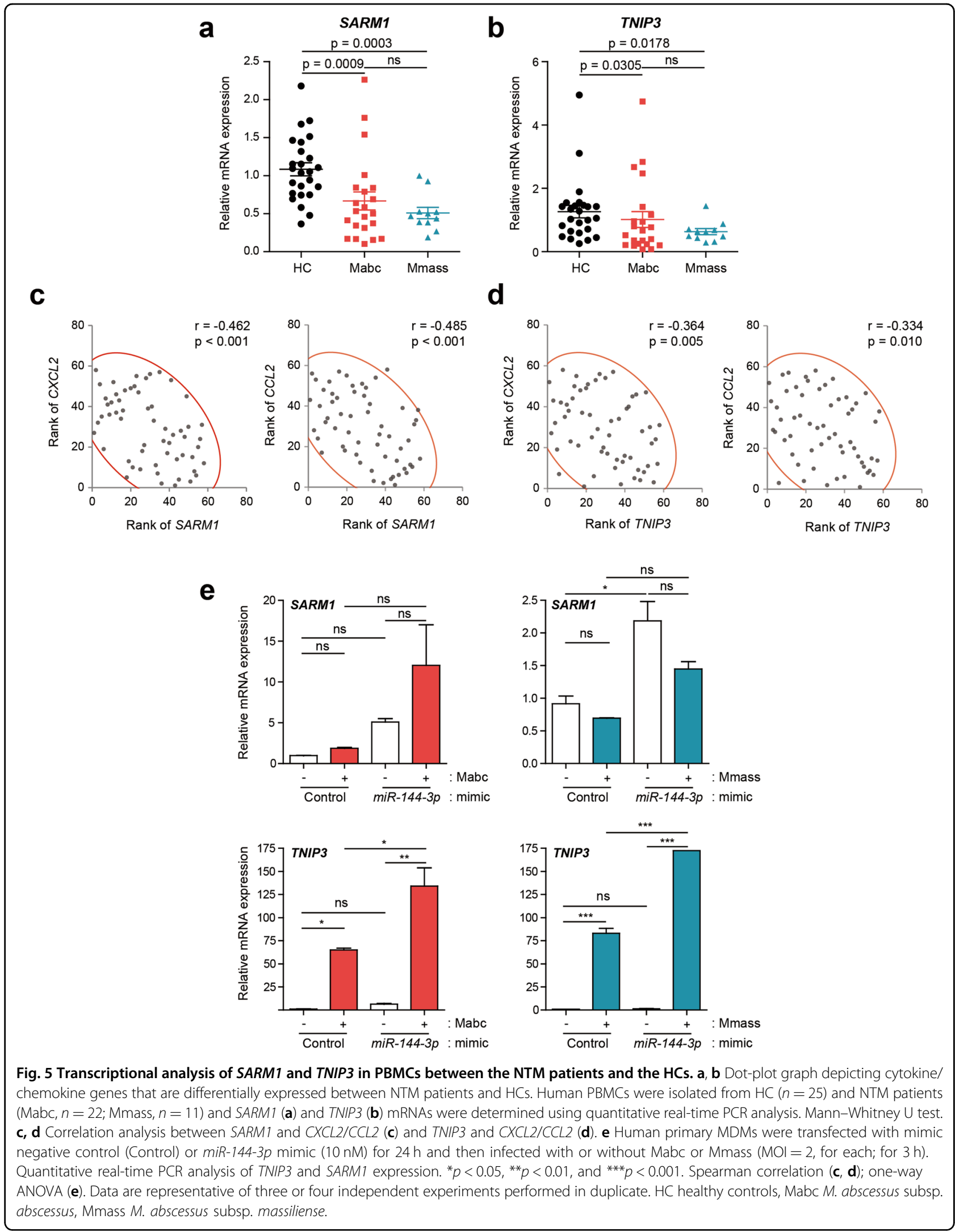




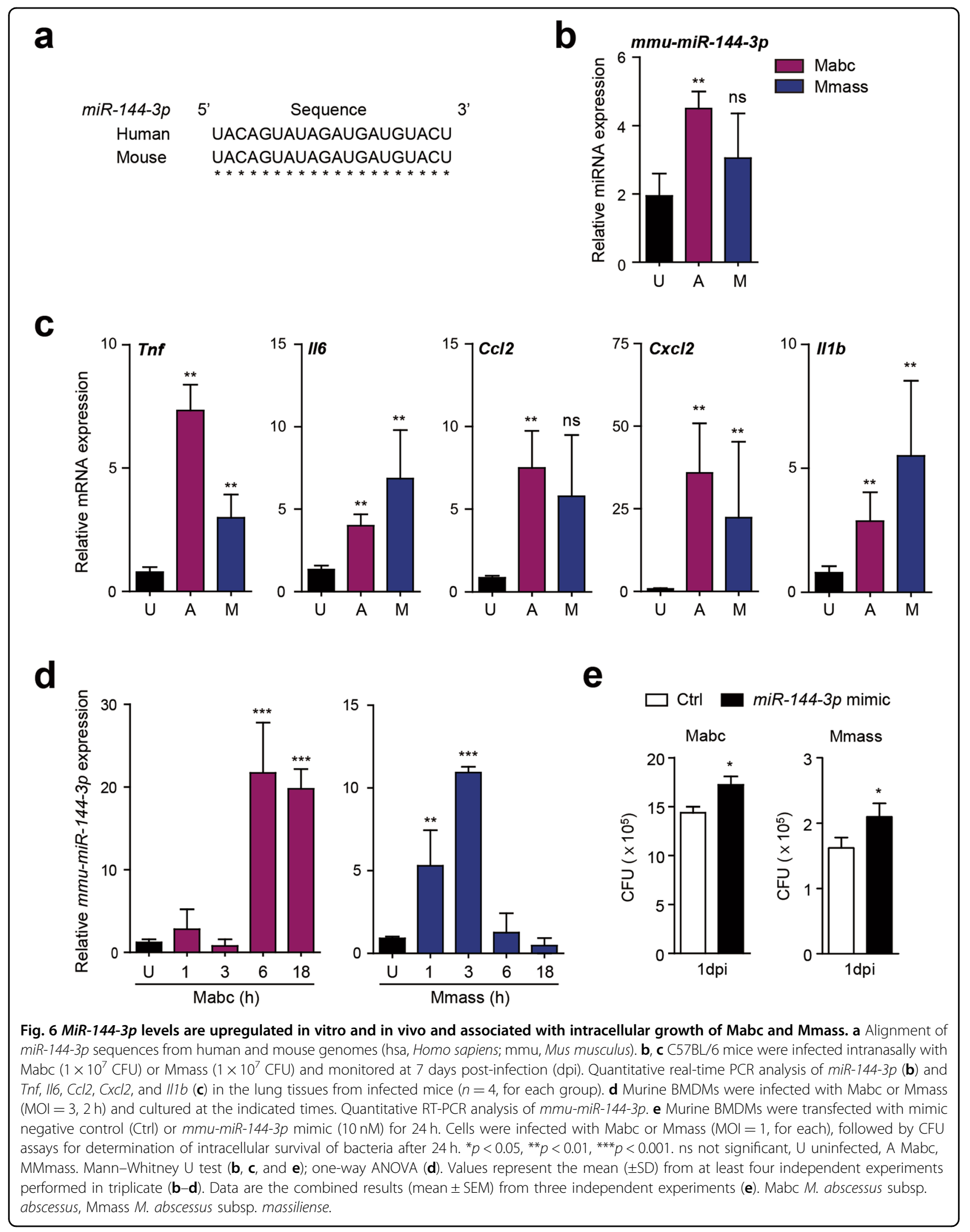


characterization of an organized pattern between immune and clinical phenotypes will be necessary for effective therapeutic interventions against human NTM infections.

MiRNAs are short noncoding RNAs and posttranscriptional regulators of a variety of biological responses, including immune responses and autophagy during $M$. tuberculosis infection ${ }^{42,43}$. Although a recent study has shown that several miRNAs can be used as potential biomarkers in NTM diseases ${ }^{44}$, the profile and role of miRNAs in human $M$. abscessus infection are unclear. Importantly, transcriptome analysis and qRTPCR validation showed that the miR-144-3p level was markedly increased in the Mabc and Mmass patients compared with the HCs. In addition, miR-144-3p was strongly correlated with the expression of several proinflammatory cytokines and chemokines, suggesting its relevance to inflammatory responses during Mabc and Mmass infection. Importantly, overexpression of $m i R$ 144-3p led to a substantial increase in the expression of proinflammatory cytokines/chemokines and the intracellular growth of Mabc and Mmass. The data partly corroborate previous studies showing that BCG infection reportedly increases miR-144-3p to inhibit autophagy and favor intracellular growth in macrophages ${ }^{45}$. Future studies are warranted to elucidate the mechanisms by which miR-144-3p contributes to promoting intracellular mycobacterial growth in the context of NTM infection.

To date, the reported levels of miR-144-3p in TB patients are heterogeneous ${ }^{46-49}$, and there have not been reports of $m i R-144-3 p$ in NTM infection. Given the finding that the miR-144-3p level is increased by NTM infection in PBMCs and mouse models in vitro and in vivo, we propose $m i R-144-3 p$ as a potential biomarker of Mabc and Mmass infection. Importantly, overexpression of miR-144-3p in human macrophages increased proinflammatory cytokine/chemokine generation in response to Mabc or Mmass infection, indicating that $m i R-144-3 p$ promotes pathological inflammation. Hsa-mir-144-3p is reportedly involved in proinflammatory responses in atopic dermatitis ${ }^{28}$. However, previous reports ${ }^{28}$ and our studies did not identify the target(s) of miR-144-3p in the context of modulation of inflammatory cytokines/chemokines. By bioinformatics analysis, we investigated two TLR negative regulators, $T N I P 3^{15}$ and $S A R M 1^{14}$, and their relationships with $m i R$ $144-3 p$, although they were not putative targets of $m i R$ 144-3p. Notably, the TNIP3 and SARM1 levels were significantly decreased in PBMCs from the Mabc-infected and Mmass-infected patients and showed a negative correlation with CXCL2 and CCL2. These data strongly indicate that depressed TNIP3 and SARM1 levels may be linked to excessive production of inflammatory chemokines in the context of $M$. abscessus infection. A deeper understanding of the key protective/pathological factors in human NTM diseases will facilitate the development of novel host-directed therapeutics for $M$. abscessus infection, which is often refractory to currently used antibiotics.

\section{Acknowledgements}

We are grateful to the late Prof. Won-Jung Koh for his guidance and encouragement to start this project. We thank Dr. H.W. Suh, S. Kim, and J.H. Choe for their excellent technical assistance. This work is supported by a grant from the Korea Health Technology R\&D Project through the Korea Health Industry Development Institute (KHIDI), funded by the Ministry of Health \& Welfare, Republic of Korea (HI20C0017), and by a National Research Foundation of Korea (NRF) grant funded by the Korean government (MSIT) (No. 2017R1A5A2015385).

\section{Author details}

'Department of Microbiology, Chungnam National University School of Medicine, Daejeon 35015, Korea. ${ }^{2}$ Department of Medical Science, Chungnam National University School of Medicine, Daejeon 35015, Korea. ${ }^{3}$ Infection Control Convergence Research Center, Chungnam National University School of Medicine, Daejeon 35015, Korea. ${ }^{4}$ School of Biological Sciences and Technology, Chonnam National University, Gwangju 61186, Korea. ${ }^{5}$ Division of Rheumatology, Regional Rheumatoid \& Degenerative Arthritis Center, Chungnam National University School of Medicine, Daejeon 35015, Korea. ${ }^{6}$ Division of Pulmonary and Critical Care, Department of Internal Medicine, Chungnam National University School of Medicine, Daejeon 35015, Korea. ${ }^{7}$ Division of Pulmonary and Critical Care Medicine, Department of Medicine, Samsung Medical Center, Sungkyunkwan University School of Medicine, Seoul 06351, Korea. ${ }^{8}$ Department of Laboratory Medicine and Genetics, Samsung Medical Center, Sungkyunkwan University School of Medicine, Seoul 06351, Korea

\section{Author contributions}

H.J.K., I.S.K., S.G.L., Y.J.K., J.Y.K., J.K.K., W.S., C.P., B.W.J., and E.K.J. participated in the research design, data curation, statistical analysis, or paper writing; Y.J.K.

participated in the animal studies; H.J.K., I.S.K., S.G.L., P.S., J.Y.K., J.K.K., W.S., and C. P. performed experiments and analysis; H.K.C., H.J.H., C.C., and B.W.J. collected blood samples from consenting subjects and clinical information; I.S.K., S.G.L., and C.P. performed the software analysis; C.P., B.W.J., and E.K.J. supervised the study or were involved in funding acquisition. All authors read and approved the final manuscript.

\section{Data availability}

The data sets used or analyzed during the current study are available from the corresponding author on reasonable request.

\section{Conflict of interest}

The authors declare that they have no conflict of interest.

\section{Publisher's note}

Springer Nature remains neutral with regard to jurisdictional claims in published maps and institutional affiliations.

Supplementary information accompanies this paper at https://doi.org/ 10.1038/s12276-020-00552-0.

Received: 4 August 2020 Revised: 3 November 2020 Accepted: 9 November 2020.

Published online: 20 January 2021

\footnotetext{
References

1. Cowman, S., van Ingen, J., Griffith, D. E. \& Loebinger, M. R. Non-tuberculous mycobacterial pulmonary disease. Eur. Respir J. 54, 1900250 (2019).

2. Koh, W. J., Stout, J. E. \& Yew, W. W. Advances in the management of pulmonary disease due to Mycobacterium abscessus complex. Int. J. Tuberc. Lung Dis. 18, 1141-1148 (2014).
} 
3. Diel, R. et al. Microbiological and clinical outcomes of treating nonmycobacterium avium complex nontuberculous mycobacterial pulmonary disease: a systematic review and meta-analysis. Chest 152 120-142 (2017).

4. Lee, M. R. et al. Mycobacterium abscessus complex infections in humans. Emerg. Infect. Dis. 21, 1638-1646 (2015).

5. Mougari, F. et al. Infections caused by Mycobacterium abscessus: epidemiology, diagnostic tools and treatment. Expert Rev. Anti Infect. Ther. 14, 1139-1154 (2016).

6. Nowag, A., Platten, M., Plum, G. \& Hartmann, P. Nontuberculous mycobacterial infections. Z. Rheumatol. 76, 752-760 (2017).

7. Brode, S. K. et al. Increased risk of mycobacterial infections associated with anti-rheumatic medications. Thorax 70, 677-682 (2015).

8. Matsuyama, M. et al. Transcriptional response of respiratory epithelium to nontuberculous mycobacteria. Am. J. Respir. Cell Mol. Biol. 58, 241-252 (2018).

9. Qian, C., Liu, J. \& Cao, X. Innate signaling in the inflammatory immune disorders. Cytokine Growth Factor Rev. 25, 731-738 (2014).

10. Muralidharan, S. \& Mandrekar, P. Cellular stress response and innate immune signaling: integrating pathways in host defense and inflammation. J. Leukoc. Biol. 94, 1167-1184 (2013).

11. Aguilar, C., Mano, M. \& Eulalio, A. MicroRNAs at the host-bacteria interface: host defense or bacterial offense. Trends Microbiol. 27, 206-218 (2019).

12. Lyu, L. et al. Small RNA profiles of serum exosomes derived from individuals with latent and active tuberculosis. Front. Microbiol. 10, 1174 (2019).

13. Sabir, N. et al. miRNAs in tuberculosis: new avenues for diagnosis and hostdirected therapy. Front. Microbiol. 9, 602 (2018).

14. Carlsson, E., Ding, J. L. \& Byrne, B. SARM modulates MyD88-mediated TLR activation through BB-loop dependent TIR-TIR interactions. Biochim. Biophys. Acta 1863, 244-253 (2016)

15. Wullaert, A. et al. LIND/ABIN-3 is a novel lipopolysaccharide-inducible inhibitor of NF-kappaB activation. J. Biol. Chem. 282, 81-90 (2007).

16. Kim, J. K. et al. MIR144* inhibits antimicrobial responses against Mycobacterium tuberculosis in human monocytes and macrophages by targeting the autophagy protein DRAM2. Autophagy 13, 423-441 (2017).

17. Kim, J. K. et al. GABAergic signaling linked to autophagy enhances host protection against intracellular bacterial infections. Nat. Commun. 9, 4184 (2018).

18. Kim, Y. S. et al. The peroxisome proliferator-activated receptor alpha-agonist gemfibrozil promotes defense against Mycobacterium abscessus Infections. Cells 9, 648 (2020).

19. R Core Team. R: A language and environment for statistical computing. $R$ Foundation for Statistical Computing, Vienna, Austria. (2013). http://www.Rproject.org/.

20. Shin, C. et al. Expanding the microRNA targeting code: functional sites with centered pairing. Mol. Cell 38, 789-802 (2010).

21. Kanehisa, M. \& Goto, S. KEGG: kyoto encyclopedia of genes and genomes. Nucleic Acids Res. 28, 27-30 (2000).

22. Mi, H., Muruganujan, A. \& Thomas, P. D. PANTHER in 2013: modeling the evolution of gene function, and other gene attributes, in the context of phylogenetic trees. Nucleic Acids Res. 41, D377-D386 (2013).

23. Borger, P., Kauffman, H. F., Postma, D. S. \& Vellenga, E. IL-7 differentially modulates the expression of IFN-gamma and IL-4 in activated human T lymphocytes by transcriptional and post-transcriptional mechanisms. J. Immunol. 156, 1333-1338 (1996).

24. Arbelaez, C. A. et al. IL-7/IL-7 receptor signaling differentially affects effector CD4+ T cell subsets involved in experimental autoimmune encephalomyelitis. J. Immunol. 195, 1974-1983 (2015).

25. Natale, M. A. et al. Trypanosoma cruzi-specific IFN-gamma-producing cells in chronic Chagas disease associate with a functional IL-7/IL-7R axis. PLoS Negl. Trop. Dis. 12, e0006998 (2018).
26. Francois, B.et al. Interleukin-7 restores lymphocytes in septic shock: the IRIS-7 randomized clinical trial. JCl Insight 3, e98960 (2018). https://doi.org/10.1172/ jci.insight.98960.

27. Kim, $\mathrm{H}$. J. et al. BACES score for predicting mortality in nontuberculous mycobacterial pulmonary disease. Am. J. Respir. Crit. Care Med. 2020. https:// doi.org/10.1164/rccm.202004-14180C

28. Dissanayake, E. et al. Hsa-mir-144-3p expression is increased in umbilical cord serum of infants with atopic dermatitis. J. Allergy Clin. Immunol. 143, 447-450 e411 (2019).

29. Lopeman, R. C., Harrison, J., Desai, M. \& Cox, J. A. G. Mycobacterium abscessus: environmental bacterium turned clinical nightmare. Microorganisms 7, 90 (2019).

30. Bryant, J. M. et al. Whole-genome sequencing to identify transmission of Mycobacterium abscessus between patients with cystic fibrosis: a retrospective cohort study. Lancet 381, 1551-1560 (2013).

31. Swenson, C., Zerbe, C. S. \& Fennelly, K. Host variability in NTM disease: implications for research needs. Front Microbiol. 9, 2901 (2018).

32. Koh, W. J. Nontuberculous mycobacteria-overview. Microbiol. Spectr. 5, (2017). https://doi.org/10.1128/microbiolspec.TNMI7-0024-2016.

33. Kwon, Y. S., Daley, C. L. \& Koh, W. J. Managing antibiotic resistance in nontuberculous mycobacterial pulmonary disease: challenges and new approaches. Expert Rev. Respir. Med. 13, 851-861 (2019).

34. Koh, W. J. et al. Clinical significance of differentiation of Mycobacterium mas siliense from Mycobacterium abscessus. Am. J. Respir. Crit. Care Med. 183 405-410 (2011).

35. Feng, Z. et al. Differential responses by human macrophages to infection with Mycobacterium tuberculosis and non-tuberculous mycobacteria. Front Microbiol. 11, 116 (2020).

36. Bernut, A. et al. Mycobacterium abscessus-induced granuloma formation is strictly dependent on TNF signaling and neutrophil trafficking. PLoS Pathog. 12, e1005986 (2016).

37. Kim, J. S. et al. Essential engagement of Toll-like receptor 2 in initiation of early protective Th1 response against rough variants of Mycobacterium abscessus. Infect. Immun. 83, 1556-1567 (2015).

38. Catherinot, E. et al. Hypervirulence of a rough variant of the Mycobacterium abscessus type strain. Infect. Immun. 75, 1055-1058 (2007).

39. Ryu, Y. J. et al. Impaired expression of Toll-like receptor 2 in nontuberculous mycobacterial lung disease. Eur. Respir. J. 30, 736-742 (2007).

40. Luther, S. A. \& Cyster, J. G. Chemokines as regulators of T cell differentiation. Nat. Immunol. 2, 102-107 (2001).

41. Pydi, S. S. et al. Down regulation of RANTES in pleural site is associated with inhibition of antigen specific response in tuberculosis. Tuberculosis $116 \mathrm{~S}$ S123-S130 (2019)

42. Rajaram, M. V., Ni, B., Dodd, C. E. \& Schlesinger, L. S. Macrophage immunoregulatory pathways in tuberculosis. Semin. Immunol. 26, 471-485 (2014).

43. Silwal, P., Kim, Y. S., Basu, J. \& Jo, E. K. The roles of microRNAs in regulation of autophagy during bacterial infection. Semin. Cell Dev. Biol. 101, 51-58 (2020).

44. Han, S. A. et al. miRNA expression profiles and potential as biomarkers in nontuberculous mycobacterial pulmonary disease. Sci. Rep. 10, 3178 (2020).

45. Guo, L. et al. MicroRNA-144-3p inhibits autophagy activation and enhances Bacillus Calmette-Guerin infection by targeting ATG4a in RAW264.7 macrophage cells. PLOS ONE 12, e0179772 (2017).

46. LV, Y. et al. Sputum and serum microRNA-144 levels in patients with tuberculosis before and after treatment. Int. J. Infect. Dis. 43, 68-73 (2016).

47. Zhou, M. et al. Circulating microRNAs as biomarkers for the early diagnosis of childhood tuberculosis infection. Mol. Med. Rep. 13, 4620-4626 (2016).

48. Wu, J. et al. Analysis of microRNA expression profiling identifies miR-155 and miR-155* as potential diagnostic markers for active tuberculosis: a preliminary study. Hum. Immunol. 73, 31-37 (2012).

49. Wang, $C$. et al. Comparative miRNA expression profiles in individuals with latent and active tuberculosis. PLOS ONE 6, e25832 (2011). 